\title{
Paper Studying and Modeling the Performance of the TCM-STBC Systems in the Rayleigh Channel
}

\author{
Mohammed Sofiane Bendelhoum ${ }^{1,2}$, Mohamed Rida Lahcene ${ }^{3,4}$, Fayssal Menezla ${ }^{2,5}$, \\ and Abderraouf Elarbi ${ }^{3}$ \\ ${ }^{1}$ Instrumentation Laboratory and Advanced Materials (ILAM), University Center Nour Bachir of El-Bayadh, Algeria \\ ${ }^{2}$ Department of Electrical Engineering, University Center Nour Bachir of El-Bayadh, Algeria \\ ${ }^{3}$ Information Processing and Telecommunication Laboratory (LTIT), Tahri Mohammed Bechar University, Algeria \\ ${ }^{4}$ Department of Technology, University Center Salhi Ahmed of Naama, Algeria \\ ${ }^{5}$ Laboratory (LEPO), Djillali Liabes University, Sidi Bel-Abbes, Algeria
}

https://doi.org/10.26636/jtit.2021.147020

\begin{abstract}
Multiple-input multiple-output (MIMO) systems will play an important role in future generations of wireless networks. Space-time block code (STBC) and space-time trellis code (STTC) are two techniques that may be used in multiantenna radio systems. This paper aims, most importantly, to study the performance of STBC systems at different values of such parameters as spectral efficiency, matrix codes and constellations. A performance comparison between STBC and STTC schemes is performed. In order to show the efficiency of the system's ability to communicate with uncoded and coded transmission structures over AWGN and Rayleigh channels, the trellis-coded modulation (TCM) is introduced. The results obtained show that the proposed TCM-STBC system model, using one and two receiving antennas, improves the performance of Rayleigh channel communication systems at $9.5 \mathrm{~dB}$ and $11.5 \mathrm{~dB}$ for a BER of $10^{-5}$.
\end{abstract}

Keywords-BER, MIMO, SNR, STBC, STTC, TCM, TCMSTBC.

\section{Introduction}

The major challenge faced by advanced communications and wireless multimedia technologies consists in achieving high performance levels with reliable communication and flexible data rates. Such outcomes are offered within a limited spectrum of bandwidths and energy consumptions rates. The MIMO technology improves spectrum efficiency and boosts channel capacity. The space-time coding (STC) technique introduces space redundancy, thanks to the addition of multiple antennas, and time redundancy thanks to channel coding. To achieve this, two prevailing space-time coding techniques are used, namely Space time block codes (STBC) and space time trellis codes (STTC). The STBC approach offers diversity gains with very low decoding complexity levels, while STTC provides both diversity and coding gains at the cost of higher decoding complexity [1].

Trellis coded modulation (TCM) is a bandwidth-efficient transmission scheme that may achieve high encoding gains by integrating encoding and modulation. A more efficient approach consists in designing trellis encoded modulation solutions in long sequences of messages [2], [3]. The permitted sequences should differ considerably from each other. The receiver may then make a decision concerning the sequences based on statistical analysis, rather than by relying on the symbol-by-symbol based computation decoding method [2].

In the literature, various studies describe the performance of such systems relying on space time coding (STC) [4], [5], STBC [6], STTC [7] and TCM [8] techniques. Other studies focused on combining STBC and STTC [9] with different systems, such as MC-CDMA [10] or OFDM [11]. In [4], a new generalized concatenated signal code construction whose inner codes are golden codes and outer codes are products of Reed-Solomon codes is proposed.

In [6], the authors proposed a new approach to decode STBC-MIMO using the Kurtosis-based blind source separation (BSS) algorithm under a quasistatic flat fading channel. The performance of a space time trellis code-based solution with the beamforming concept and relying on various modulation techniques is analyzed in [7]. Hashem Ali Khan et al. [8] analyzed signal constellations based on orthogonal space-time codes (OSTCs), which are theoretically suited for MIMO systems. BER performance of STBC and STTC in the MIMO satellite channel is studied by Jing and $\mathrm{Wu}$ [9]. In [10], balanced STTC (B-STTC)based STBC site diversity is proposed to improve the performance of MC-CDMA systems by mitigating multi-cell interference and is extended to the STBC-based B-STTC site diversity technique. STBC-OFDM techniques are studied in [11] for wireless channels using BPSK modulation and maximum likelihood detection for two transmit and two receive antennas.

Our contribution consists in proposing a combination of two systems: trellis coded modulation (TCM) and the space-time block code (STBC) for improving performance 
of transmission. The remaining part of the paper is organized as follows.

In Section 2, space time block coding is introduced. Section 3 presents a comparison between STBC and STTC schemes. Section 4 describes performance of communication with uncoded and coded transmission systems, using the TCM structure. Section 5 discusses the results of simulations of the proposed scheme, using TCM code modulation concatenated with the STBC. Finally, the paper concludes by presenting certain insights into the prospects concerning our present work.

\section{Space Time Block Coding}

STBC stipulates that each block is coded using predetermined rules, independently of the other blocks. A decoding error occurring in one block does not affect other blocks. This pace-time diversity method has been proposed by Alamouti [12] using transmit and receive antennas. This method was then generalized by Tarokhet for $n$ transmit antennas and $m$ receive antennas in [13], [14]. Figure 1 illustrates the communication system of the original Alamouti STBC solution using two transmit and two receive antennas. The idea is to send a block of symbols via several transmit antennas. The symbols sent during the duration of a block vial all the antennas are orthogonal. Real or complex symbols may be used for the transmission. The transmit and receive antennas are positioned in such a way that there is no correlation between them. The channels between each pair of transmit and receive antennas are independent and uncorrelated under these conditions. Due to the orthogonality of symbols, it is possible to use a simple linear recombination algorithm at the reception side to recover the symbols transmitted by all the antennas.

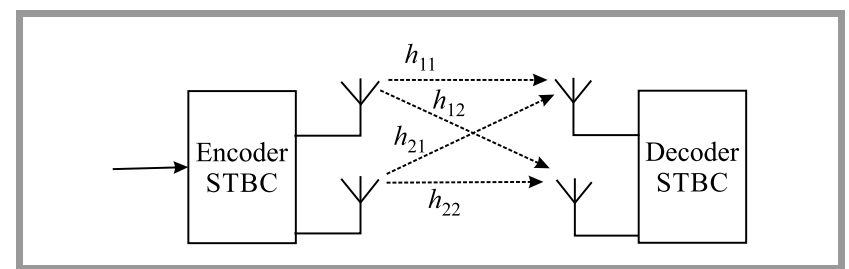

Fig. 1. Alamouti's STBC communication system.

Information symbols will be processed in pairs while generating four other symbols, two per each antenna [12]. Two signals are transmitted simultaneously from both antennas at time $t$. The signal transmitted by antenna one is defined by $x_{0}$ and the one transmitted by antenna two is defined

Table 1

Transmission sequence for two antennas

\begin{tabular}{|c|c|c|}
\hline Temps & Antenna 1 & Antenna 2 \\
\hline \hline$t$ & $x_{0}$ & $x_{1}$ \\
\hline$t+T$ & $-x_{1}^{*}$ & $x_{0}^{*}$ \\
\hline
\end{tabular}

by $x_{1}$. At time $t+T$, the signal $\left(-x_{1}^{*}\right)$ is transmitted by antenna one and signal $x_{0}^{*}$ is transmitted by antenna two, according to Table 1 .

At time $t$, the signal received at antenna $j$ is given by:

$$
r_{t}^{j}=\sum_{i=1}^{2} h_{i j} x_{t}^{i}+n_{t}^{j}
$$

The diversity matrixes $G_{M}$ and $H_{M}$, are given by [12], [15]:

$$
\begin{aligned}
& G_{2}=\left[\begin{array}{cc}
x_{1} & -x_{2}^{*} \\
x_{2} & x_{1}^{*}
\end{array}\right] \text {, } \\
& G_{3}=\left[\begin{array}{cccccccc}
x_{1} & -x_{2} & -x_{3} & -x_{4} & x_{1}^{*} & -x_{2}^{*} & -x_{3}^{*} & -x_{4}^{*} \\
x_{2} & x_{1}^{*} & x_{4} & -x_{3} & x_{2}^{*} & x_{1}^{*} & x_{4}^{*} & -x_{3}^{*} \\
x_{3} & -x_{4} & x_{1} & x_{2} & x_{3}^{*} & -x_{4}^{*} & x_{1}^{*} & x_{2}^{*}
\end{array}\right]^{T}, \\
& G_{4}=\left[\begin{array}{cccccccc}
x_{1} & -x_{2} & -x_{3} & -x_{4} & x_{1}^{*} & -x_{2}^{*} & -x_{3}^{*} & -x_{4}^{*} \\
x_{2} & x_{1}^{*} & x_{4} & -x_{3} & x_{2}^{*} & x_{1}^{*} & x_{4}^{*} & -x_{3}^{*} \\
x_{3} & -x_{4} & x_{1} & x_{2} & x_{3}^{*} & -x_{4}^{*} & x_{1}^{*} & x_{2}^{*} \\
x_{4} & x_{3} & -x_{2} & x_{1} & x_{4}^{*} & x_{3}^{*} & -x_{2}^{*} & x_{1}^{*}
\end{array}\right]^{T}, \\
& H_{4}=\left[\begin{array}{cccc}
x_{1} & -x_{2}^{*} & \frac{x_{3}^{*}}{\sqrt{2}} & \frac{x_{3}^{*}}{\sqrt{2}} \\
x_{2} & x_{1}^{*} & \frac{x_{3}^{*}}{\sqrt{2}} & \frac{x_{3}^{*}}{\sqrt{2}} \\
\frac{x_{3}}{\sqrt{2}} & \frac{x_{3}}{\sqrt{2}} & \frac{-x_{1}-x_{1}^{*}+x_{2}-x_{2}^{*}}{2} & \frac{-x_{1}-x_{1}^{*}+x_{2}+x_{2}^{*}}{2} \\
\frac{x_{3}}{\sqrt{2}} & \frac{-x_{3}}{\sqrt{2}} & \frac{-x_{1}-x_{1}^{*}+x_{2}-x_{2}^{*}}{2} & \frac{-x_{1}-x_{1}^{*}+x_{2}+x_{2}^{*}}{2}
\end{array}\right]^{T}
\end{aligned}
$$

The maximum likelihood decoder chooses a pair of signals. For two transmit G2 codes (Alamouti), the technique for combining $x_{0}$ and $x_{1}$ is [12]:

$$
\left\{\begin{array}{l}
\tilde{x}_{0}=\sum_{j=1}^{m}\left[r_{1}^{j} h_{1 j}^{*}+\left(r_{2}^{j}\right)^{*} h_{2 j}\right] \\
\tilde{x}_{1}=\sum_{j=1}^{m}\left[r_{1}^{j} h_{2 j}^{*}-\left(r_{2}^{j}\right)^{*} h_{1 j}\right]
\end{array} .\right.
$$

For two receive antennas:

$$
\left\{\begin{array}{l}
\tilde{x}_{0}=r_{1}^{1} h_{11}^{*}+\left(r_{2}^{1}\right)^{*} h_{21}+r_{1}^{2} h_{12}^{*}+\left(r_{2}^{2}\right)^{*} h_{22} \\
\tilde{x}_{1}=r_{1}^{1} h_{21}^{*}-\left(r_{2}^{1}\right)^{*} h_{11}+r_{1}^{2} h_{22}^{*}-\left(r_{2}^{2}\right)^{*} h_{12}
\end{array},\right.
$$

where $h_{11}, h_{12}, h_{21}$ and $h_{22}$ represent the corresponding complex time domain channel transfer factors.

Figure 2 shows simulation results obtained based on the STBC scheme at $1 \mathrm{bps}$, using one, two, three, and four transmitting antennas and one receiving antenna. The output signal power is transmitted in whole when using one antenna. Otherwise, it will be equally divided when using multiple antennas [15]. The transmission performed while using one, two transmitting antennas relies on a BPSK constellation, as well as on the G1 (SISO model) and code G2. For three and four transmit antennas, the constellation used is QPSK with codes G3 and G4, for a spectral efficiency of $1 \mathrm{bps}$. For a BER of $10^{-5}$ a significant 


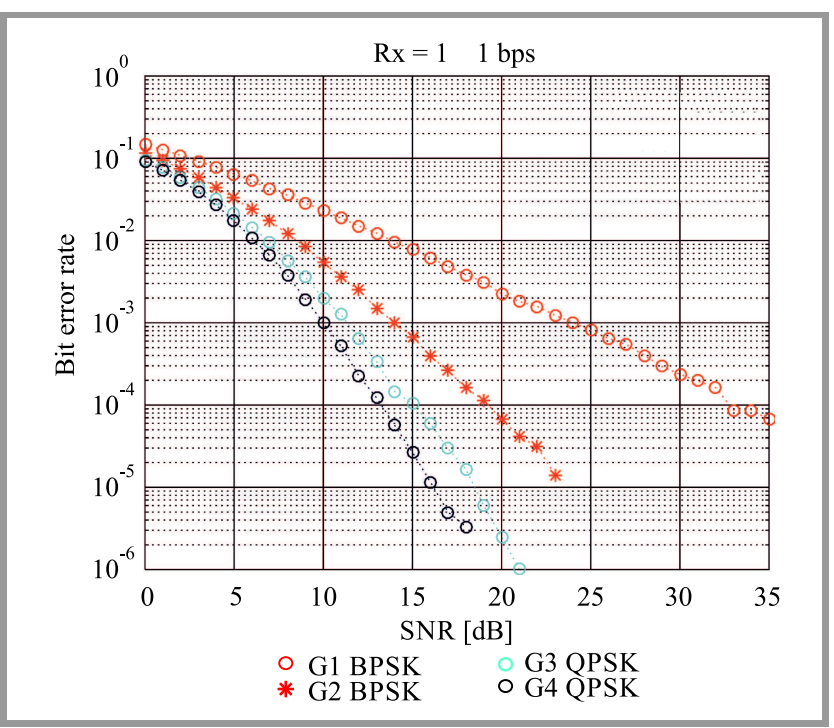

Fig. 2. BER versus SNR for different STBC codes for 1 bps with only one receiving antenna.

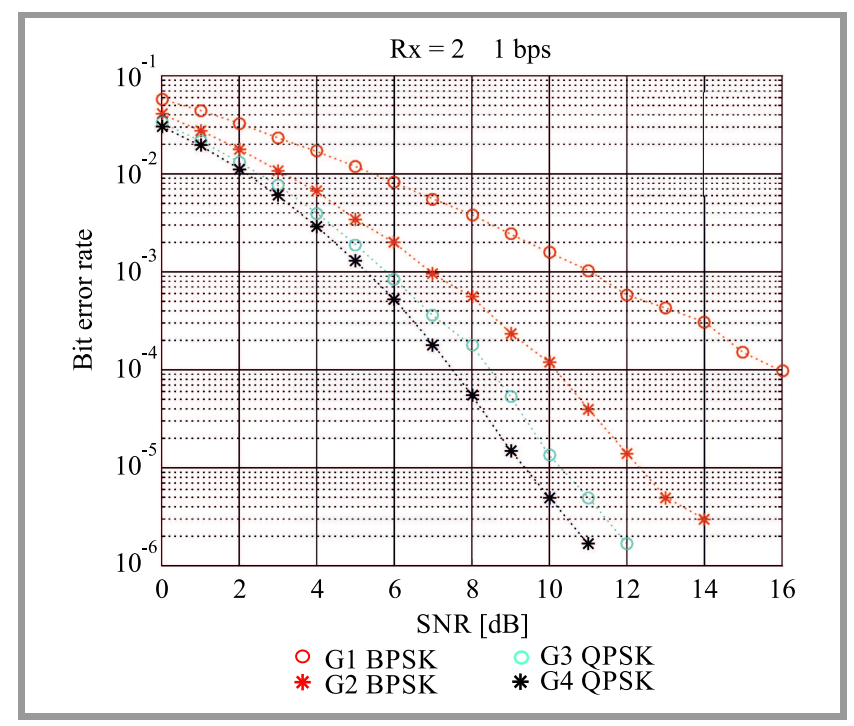

Fig. 3. BER versus SNR for different STBC codes for 1 bps with two receiving antennas.

improvement in performance is observed, equaling approx. $7.5 \mathrm{~dB}$ for code $\mathrm{G} 4$, compared to code $\mathrm{G} 2$ for a single receiving antenna. If the number of receiving antennas is increased, this gain equals $3 \mathrm{~dB}$, as shown in Fig. 3, with spectral efficiency amounting to 2 bps. In Fig. 3, the G1 variant is called SIMO.

Figure 4 depicts the simulation results obtained for $2 \mathrm{bps}$ using one, two, three, and four transmit antennas. The transmission is performed using one, two transmit antennas with the QPSK constellation, as well as code G1 and G2. For three and four transmit antennas, the 16-QAM constellation is used with codes G3 and G4, respectively. It is obvious that code G4 offers a gain of approx. $5 \mathrm{~dB}$, compared to code $\mathrm{G} 2$ for a of $10^{-5}$ and one receiving antenna.

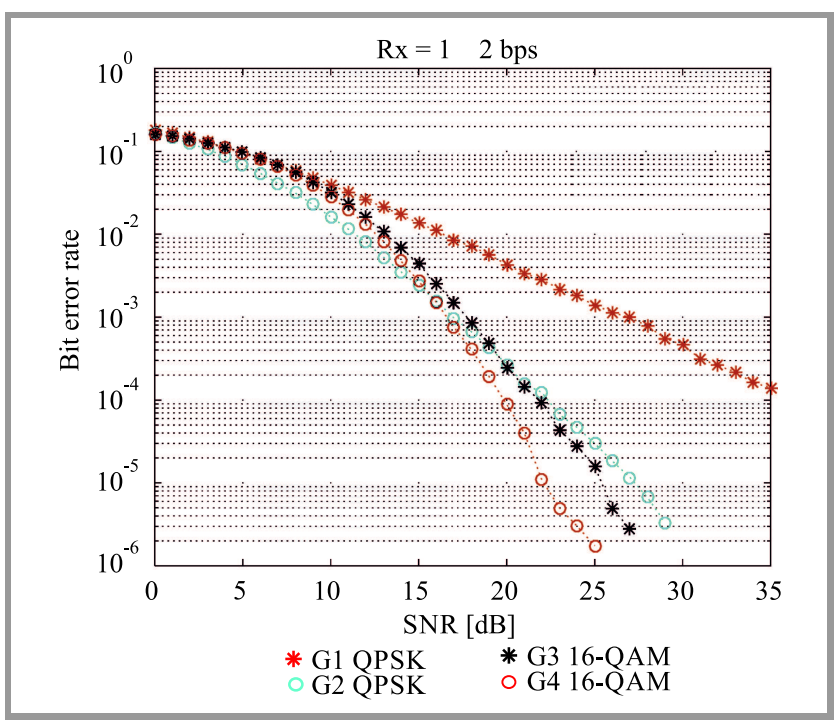

Fig. 4. BER versus SNR for different STBC codes for 2 bps with a single receiving antenna.

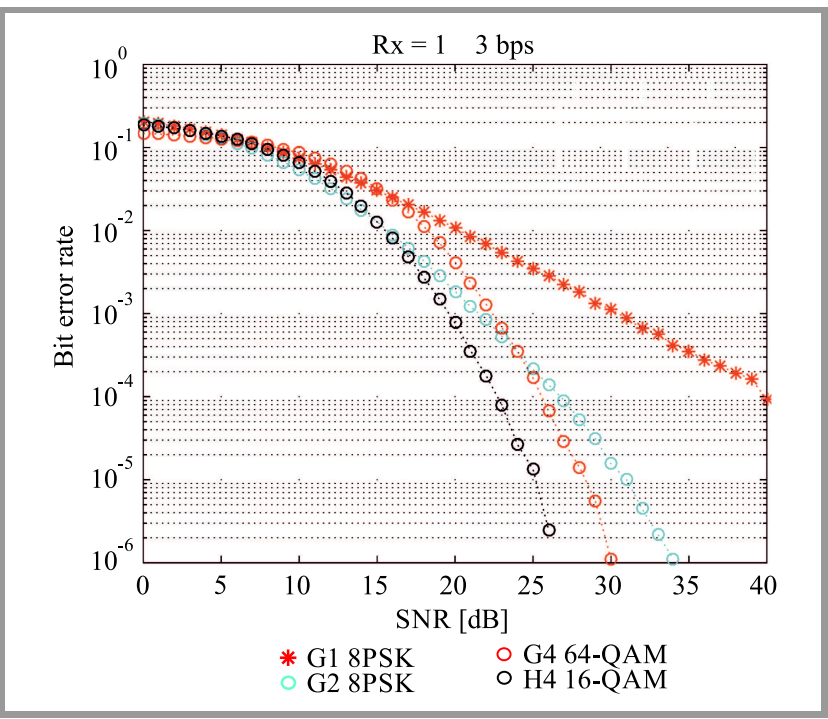

Fig. 5. BER versus SNR for different STBC codes for 3 bps with only one receiving antenna.

BER results versus SNR for 3 bps are shown in Fig. 5. For the 8-PSK modulation, codes G1 and G2 are used. Code G4 is used for the 64-QAM modulation. The 16-QAM modulation is used with code H4. Figure 4 clearly illustrates that the use of spatial diversity improves performance of the transmission system. Thus, there is an improvement of about $6 \mathrm{~dB}$ using code $\mathrm{H} 4$, compared to code $\mathrm{G} 2$ for BER of $10^{-5}$ for one receiving antenna.

\section{Space Time Trellis Codes}

STTCs combine modulation and trellis coding for transmissions across multiple transmit antennas [16], [17]. The number of antennas may be increase without much complexity. Two symbols are simultaneously emitted from 
these two antennas for each path in the trellis accordingly. Indices corresponding to the emitted symbols are represented in front of each line, indicating the state in the elementary lattice. These indices are ordered from left to right, considering the disposition of the segments, clockwise for each state. The number of the grouped indices is equal to the number of antennas.

The space time trellis encoder transmits symbols $x_{k, 1}$ and $x_{k, 2}$ over two transmitter antennas $T_{x 1}$ and $T_{x 2}$. For each instant $k$, in a 4-state scenario with 4PSK constellation, the output symbols are given by [15], [17]:

$$
\begin{aligned}
& x_{k, 1}=0 . d_{k, 1}+0 . d_{k, 2}+1 . d_{k-1,1}+2 . d_{k-1,2}, \\
& x_{k, 2}=1 . d_{k, 1}+2 . d_{k, 2}+0 . d_{k-1,1}+0 . d_{k-1,2},
\end{aligned}
$$

where $d_{k, i}$ represents current input bits and $d_{k-1, i}$ represents previous input bits and $i=1,2$.

For each transmission time, the space time trellis decoder uses two 4-state receiver antennas with 4PSK. Symbols $x_{1}$ and $x_{2}$ are transmitted by antennas $T_{x 1}$ and $T_{x 2}$. At the reception antennas $R_{x 1}$ and $R_{x 2}$, we have:

$$
\begin{aligned}
& y_{1}=h_{11} x_{1}+h_{12} x_{2}+n_{1}, \\
& y_{2}=h_{21} x_{1}+h_{22} x_{2}+n_{2} .
\end{aligned}
$$

The corresponding complex time-domain channel transfer factors are represented by $h_{11}, h_{12}, h_{21}$ and $h_{22}$. The $n_{1}$ and $n_{2}$ are independent complex valued AWGNs having a zero mean and a power spectral density of $N_{0} / 2$ per dimension at time $k$ and $k+1$, respectively.

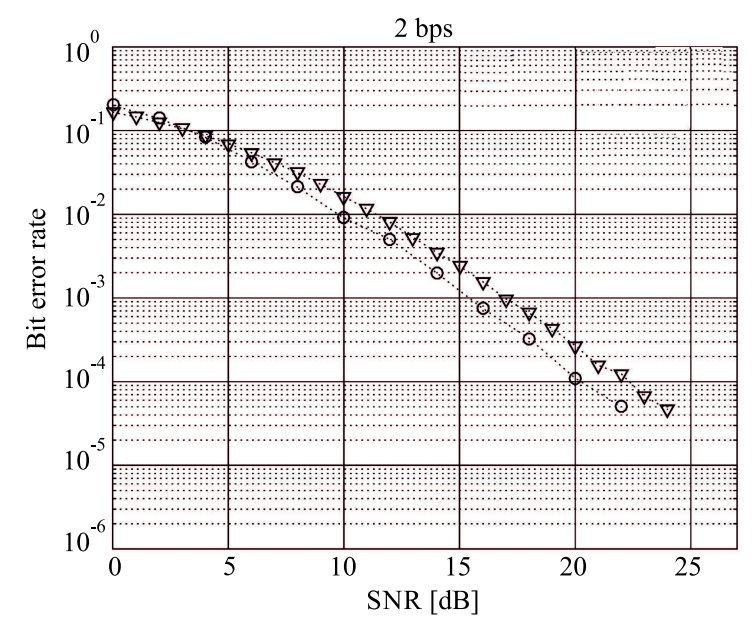

o STTC 16 state QPSK $\nabla$ STBC G2 QPSK

Fig. 6. BER versus SNR for 2 bps with one receiving antenna.

Figure 6 illustrates a comparison between STBC and STTC schemes for $2 \mathrm{bps}$, using one receive antenna. In STBC (code G2) and STTC (16-state codes), we use two transmit antennas with the QPSK constellation. The output signal power is transmitted fully when using one antenna. Otherwise, it will be equally divided when using multiple antennas.

Figure 6 clearly depicts that space-time trellis codes generate better results than the block space-time coding. For a BER of $5 \cdot 10^{-5}$, the STTC provides a gain of $2 \mathrm{~dB}$ over the STBC scheme.

Figure 7 shows the simulation results for 3 bps using one receiving antenna for the STBC scheme (code G2) and STTC scheme (16 states). Two transmit antennas with the 8-PSK constellation are used. For a BER of $3 \cdot 10^{-5}$, STTC provides a $3.4 \mathrm{~dB}$ gain over the STBC scheme.

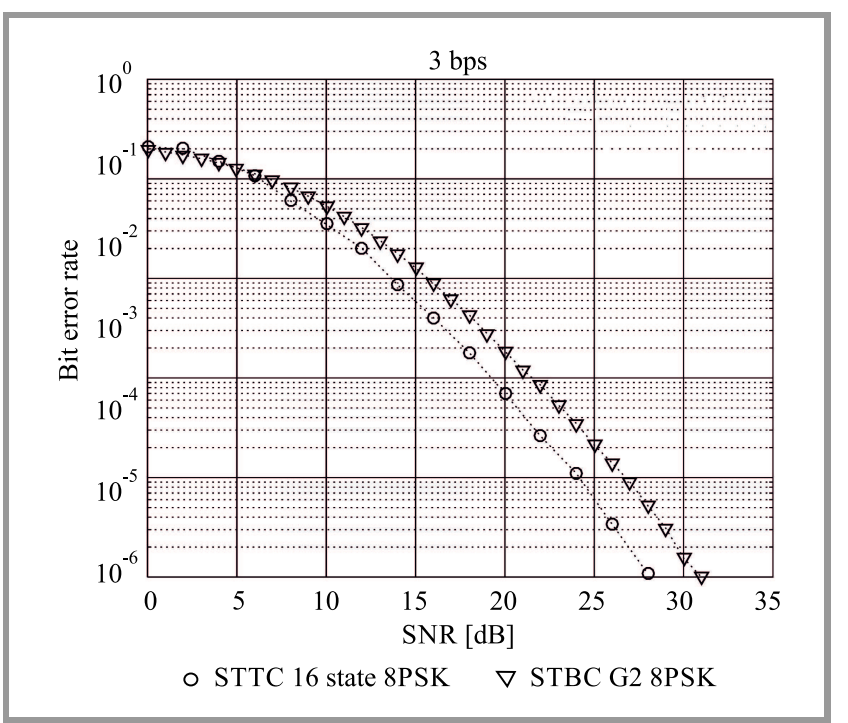

Fig. 7. BER versus SNR for spectral efficiency of 3 bps with only one receiving antenna.

Both block space time codes and space time trellis codes are characterized by broadcast diversity. Space-time codes in blocks are constructed from orthogonal matrixes and may all exploit diversity. They are easily decodable by a maximum likelihood decoder and linear processing at the reception, but they suffer from a lack of coding gain. On the other hand, the lattice space-time code has both diversity and coding gain, but it is complex to decode.

\section{Trellis Coded Modulation}

Trellis coded modulation (TCM) is one of the coded modulation techniques [18] used in MIMO systems. It combines the choice of a modulation scheme with that of a convolutional code, in order to obtain immunity against noise. More specifically, transmission is performed without increasing the bandwidth of the transmitted signal [19]. TCM is used in many modern transmission systems, such as modems.

For the 8-state TCM encoder with a spectral efficiency of $2 \mathrm{bps}$, the generating matrixes are [20]:

$$
G=\left(\begin{array}{ccc}
0 & 0 & 1 \\
1 & 0 & 0 \\
0 & 1 & 0
\end{array}\right), \quad T=\left(\begin{array}{ll}
0 & 0 \\
1 & 0 \\
0 & 1
\end{array}\right) .
$$

Matrix $G$ is obtained by the dependence between each memory of the convolutional coder and the other memories. Matrix $T$ is based on the dependence of the coder memory on coder inputs. The generator polynomial is: 


$$
P=(11,02,04)_{8}
$$

In Fig. 8, performance of the TCM scheme for 8-PSK modulation over the AWGN channel and Rayleigh channel is shown. The rate of this code is $R=2 / 3$. It is clear from this figure that for a Gaussian channel, the gain provided by the TCM scheme is about $2 \mathrm{~dB}$ for a BER of $10^{-5}$ and that this gain is $9 \mathrm{~dB}$ for a BER of $10^{-3}$ over a Rayleigh channel.

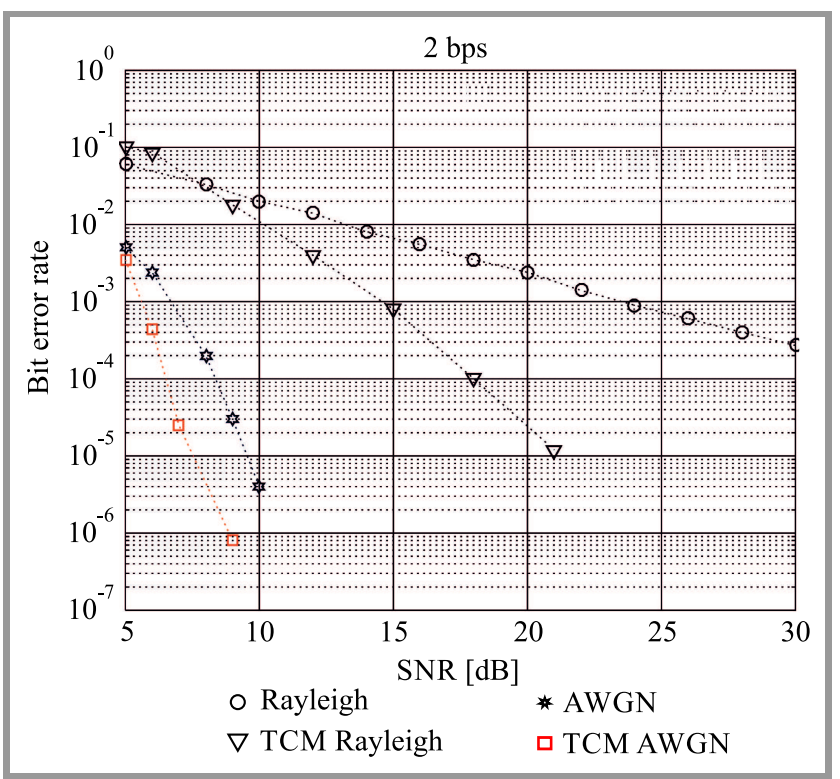

Fig. 8. Performance of TCM code in AWGN and Rayleigh channel.

Figure 8 illustrates that the use of TCM code modulation offers considerable gains in coding compared to an uncoded transmission system.

\section{Association between TCM and STBC}

In this section, the proposed scheme, named TCM-STBC, is presented. Figure 9 presents a block diagram of the scheme using TCM code modulation concatenated with STBC.

The following are the different steps needed to transmit $\mathrm{K}$ bits (data source) using TCM-STBC:

1. In the TCM block, we choose $N$-states and the type of generator polynomial $P$, where the rate is: $\operatorname{rate}_{T C M}=$ $\frac{K}{(K+1)}$.

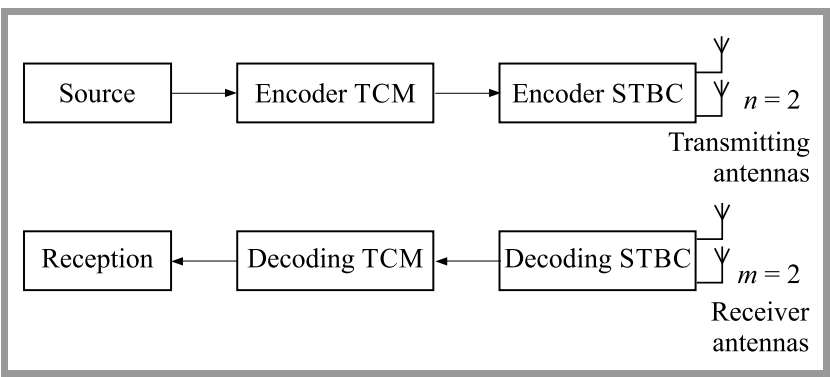

Fig. 9. Transmission system for STBC associated with TCM.
2. The number of bits at the output of the TCM block is equal to $K+1$ bits.

3. The resulting $K+1$ bits of step 2 are injected in the STBC block. Then, the STBC block rate will be rate $_{S T C B}$, depending on $G_{M}$ or $H_{M}$ matrixes and the constellation type.

4. The resulting rate of the entire TCM-STBC system will be: $\operatorname{rate}_{T C M}-S T B C=\operatorname{rate}_{T C M} \times$ rate $_{S T B C}$.

5. The output signal power is totally transmitted in whole when using one antenna. Otherwise, it will be equally divided when using multiple antennas.

6. At the receiver part, we applied the STBC and TCM decoding method.

Finally, we receive the transmitted data.

To enable a performance analysis of the proposed TCMSTBC scheme, Fig. 10 illustrates the impact of memory size (number of states) after the concatenation of the TCM with the STBC for 8-PSK modulation and the generator polynomial is $P=(11,02,04)_{8}$ with spectral efficiency of 2 bps and $2 / 3$. We can notice that when increasing the number of states for the TCM coder, performance of BER, as a function of the SNR performance, improves. For example, for a BER of $10^{-4}$, the 16-state TCM-STBC provides a gain of about $1 \mathrm{~dB}$ over the 8-state TCM-STBC.

Comparison between TCM-STBC and STTC is performed for the same memory size. Since STTC offers comparable results for 16 and 32 states, the comparison is performed for 16 states. For spectral efficiency of $2 \mathrm{bps}$, TCM-STBC uses the 8-PSK modulation and the generator polynomial is $P=(11,02,04)_{8}$ with a rate of $2 / 3$. For STTC, QPSK modulation in the Rayleigh channel is used.

Figure 11 shows a comparison between the performance of TCM-STBC (code G2) and STTC for one receiving antenna. It is clear that the gain generated by the use of TCM-STBC is considerable. For a BER of $10^{-4}$ gain associated with STBC is $10.5 \mathrm{~dB}$ relative to STTC in the Rayleigh channel.

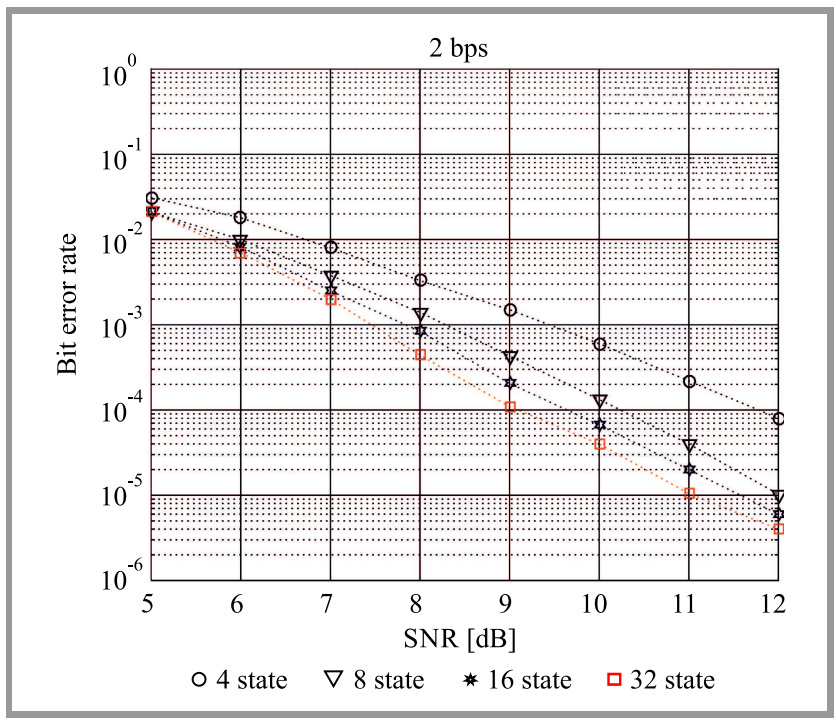

Fig. 10. Performance of TCM-STBC with 4, 8, 16 and 32 states. 


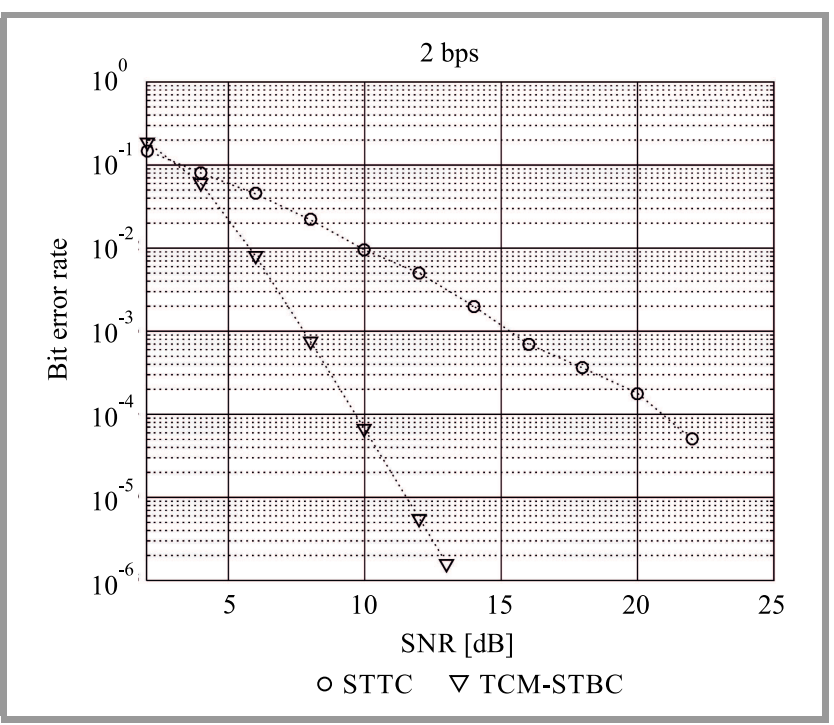

Fig. 11. Comparison between STTC and TCM-STBC proposed.

The results obtained illustrate the effectiveness of using TCM coding, as it offers a gain compared to STBC, thus leading to a diversity gains, unlike in the case of STTC which uses both coding and diversity gains.

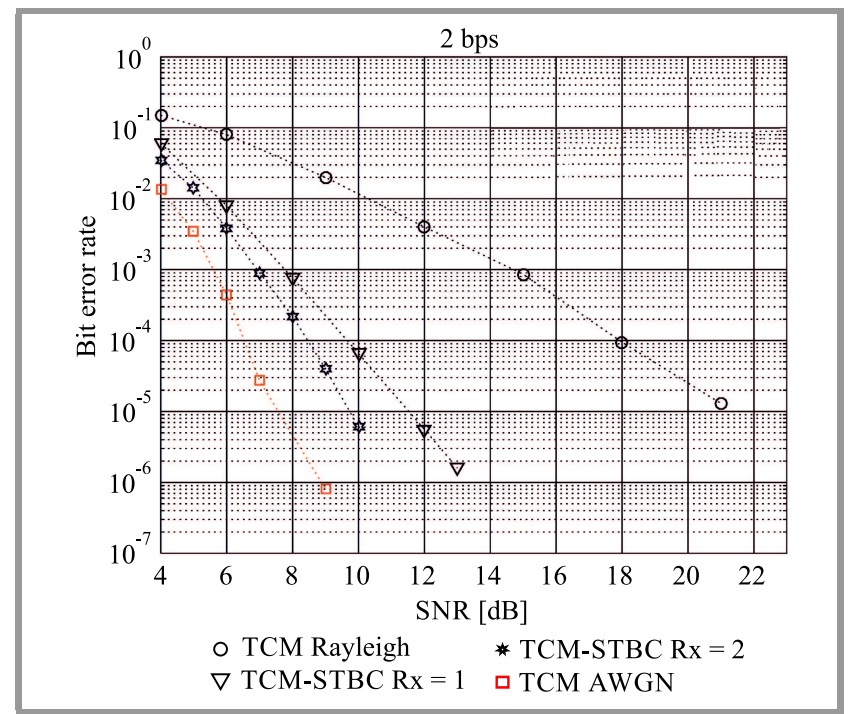

Fig. 12. Performance of TCM-STBC for one and two receiving antennas.

Figure 12 presents the effect of using two receive antennas for the TCM-STBC model (code G2) with 8-PSK modulation, spectral efficiency of 2 bps and a $2 / 3$ rate in the Rayleigh channel. It is very clear that the curves showing BER versus SNR obtained with the use of TCM-STBC are well below the TCM curve in the Rayleigh channel. The gain is $9.5 \mathrm{~dB}$ for a single receiving antenna and $11.5 \mathrm{~dB}$ for two receiving antennas, for BER of $10^{-5}$. These curves are much closer to the curve obtained with the use of the TCM system in the AWGN channel. Indeed, the addition of a receiving antenna to the proposed TCM-STBC model improves the performance parameters concerned.

\section{Conclusion}

This paper focuses on multi-antenna radio systems that may be implemented in future generations of wireless networks. Space time block codes (STBC) with different antenna systems are presented and evaluated. BER performance of STBC and space time trellis code (STTC) solutions is compared using different configurations. The authors prove that the TCM scheme implemented over AWGN and Rayleigh channels may significantly improve performance of the transmission system.

Simulation results clearly show that the scheme we have proposed, relying on TCM code modulation and concatenated with the STBC system, significantly increases transmission performances. An increase in the number of encoder states generates additional gains, but significantly increases the calculation lead time during decoding. Moreover, addition of receiving antennas makes it possible to increase the efficiency of TCM associated with the STBC structure. This leads to a gain of $9.5 \mathrm{~dB}$ with one receiver antenna and $11.5 \mathrm{~dB}$ with two receiver antennas in the Rayleigh channel, and reduces SNR.

\section{References}

[1] J. K. Daksh, R. Mohan, and S. Sharma, "Performance analysis with space-time coding in MIMO-OFDM Systems with multiple antennas", Int. J. of Adv. Comp. Res., vol. 3, no. 2, issue 10, 2013 [Online]. Available: https://www.accentsjournals.org/PaperDirectory/Journal/ IJACR/2013/6/17.pdf

[2] R. Vithiya, V. Karthika, P. Samundeeswari, and S. Mohanraj, "Trellis coded modulation for MIMO wireless communication system", Int. J. of Engin. Res. and Technol., vol. 2, no. 12, pp. 2577-2583, 2013 [Online]. Available: https://www.ijert.org/research/ trellis-coded-modulation-for-mimo-wireless-communication-systemIJERTV2IS120927.pdf

[3] Y. Wu, Y. Liu, H.-C. Chang, Y.-C. Liao, and H.-C. Chang, "Earlypruned k-best sphere decoding algorithm based on radius constraints", in Proc. IEEE Int. Conf. on Commun. ICC 2008, Beijing, China, 2008, pp. 4496-4500 (DOI: 10.1109/ICC.2008.843).

[4] A. A. Kreshchuk and V. V. Zyablov, "Generalized concatenated system with embedded space-time codes for MIMO systems", J. of Commun. Technol. and Electron., vol. 59, no. 12, pp. 1489-1500, 2014 (DOI: 10.1134/S1064226914120109).

[5] E. Telatar, "Capacity of multi-antenna Gaussian channels", Eur. Trans. on Telecommun., vol. 10, no. 6, pp. 585-595, 1999 (DOI: 10.1002/ett.4460100604).

[6] S. A. K. Alrufaiaat and A. Q. J. Althahab, "Robust decoding strategy of MIMO-STBC using one source Kurtosis based GPSO algorithm", J. of Ambient Intell. and Human. Comput., 2020 (DOI: 10.1007/s12652-020-02288-1).

[7] S. R. Chopra, A. Gupta, and H. Monga, "Performance analysis of space time trellis codes in Rayleigh fading channel", in Harmony Search and Nature Inspired Optimization Algorithms, N. Yadav et al., Eds. Advances in Intelligent Systems and Computing, vol. 741, pp. 957-967. Springer, 2019 (DOI: 10.1007/978-981-13-0761-4_90).

[8] M. Hashem Ali Khan, T. Shin, M. H. Lee, and J.-G Chung, "Signal constellations of quasi-orthogonal space-time codes for MIMO systems", Wirel. Pers. Commun., vol. 85, no. 4, pp. 2003-2019, 2015 (DOI: 10.1007/s11277-015-2887-z).

[9] Q. Jing and J. Wu, "Performance comparison of space-time block and trellis codes in the MIMO land mobile satellite channels", $R a$ dioelectron. and Commun. Syst., vol. 60, no. 1, pp. 3-17, 2017 (DOI: 10.3103/S0735272717010010). 
[10] M. Pushpakodi and N. Kumaratharan, "BER enhancement of MCCDMA through B-STTC based STBC and STBC based B-STTC site diversity techniques", Universal J. of Commun. and Netw., vol. 1, no. 3, pp. 81-87, 2013 (DOI: 10.13189/ujcn.2013.010301).

[11] H. A. Bakir, F. Debbat, and F. T. Bendimerad, "Performance comparison of STBC-OFDM with maximum likelihood detection for Rayleigh fading channel", Int. J. of Engin. Res. in Africa, vol. 26, pp. 111-121, 2016 (DOI: 10.4028/www.scientific.net/JERA.26.111).

[12] S. M. Alamouti, "A simple transmitter diversity technique for wireless communications", IEEE J. on Selec. Areas in Commun., vol. 16, no. 8, pp. 1451-1458, 1998 (DOI: 10.1109/49.730453).

[13] V. Tarokh, H. Jafarkhani, and A. R. Calderbank, "Space-time block codes from orthogonal designs", IEEE Trans. on Inform. Theory, vol. 45, no. 5, pp. 1456-1467, 1999 (DOI: 10.1109/18.771146).

[14] V. Tarokh, H. Jafarkhani, and A. R. Calderbank, "Space-time block coding for wireless communications: Performance results", IEEE J. on Selec. Areas in Commun., vol. 17, no. 3, pp. 451-460, 1999 (DOI: 10.1109/49.753730).

[15] L. L. Hanzo, T. H. Liew, B. L. Yeap, R. Y. S. Tee, and S. X. Ng, Turbo Coding, Turbo Equalisation and Space-Time Coding, 2nd ed. Wiley, 2011 (ISBN: 9780470978337).

[16] A. Wittneben, "Base station modulation diversity for digital simulcast", in Proc. 41st IEEE Veh. Technol. Conf. VTC, St. Louis, MO, USA, 1991, pp. 848-853 (DOI: 10.1109/VETEC.1991.140615).

[17] V. Tarokh, N. Seshadri, and A. R. Calderbank, "Space-time codes for high data rate wireless communication: Performance criterion and code construction", IEEE Trans. on Inform. Theory, vol. 44, no. 2, pp. 744-765, 1998 (DOI: 10.1109/18.661517).

[18] G. Ungerboeck, "Channel coding with multilevel/phase signals", IEEE Trans. on Inform. Theory, vol. 28, no. 1, pp. 55-67, 1982 (DOI: 10.1109/TIT.1982.1056454).

[19] L. F. Wei, "Trellis-coded modulation with multidimensional constellations", IEEE Trans. on Inform. Theory, vol. 33, no. 4, pp. 483-501, 1987 (DOI: 10.1109/TIT.1987.1057329).

[20] P. Robertson and T. Worz, "Bandwidth-efficient turbo trellis-coded modulation using punctured component codes", IEEE J. on Selec. Areas in Commun., vol. 16, no. 2, pp. 206-218, 1998 (DOI: 10.1109/49.661109).

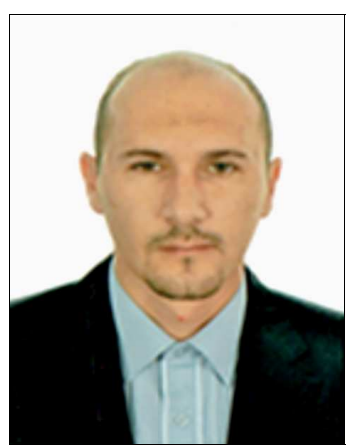

Mohammed Sofiane Bendelhoum obtained his B.Eng. degree in Biomedical Electronics and M.Sc. in Signals and Systems from Tlemcen University. He also earned his Ph.D. from Sidi Bel Abbes University, Algeria. Since 2014, he has been an Associate Professor at the University Center of El-Bayadh and performs his research at the Instrumentation Laboratory and Advanced Materials University Center. His research interests are in image processing, medical image compression, wavelets transform, turbo encoding, turbo equalization, wireless communication systems and signal processing.

E-mail: Bendelhoum_med@yahoo.fr

Instrumentation Laboratory

and Advanced Materials (ILAM)

University Center Nour Bachir of El-Bayadh

El-Bayadh, Algeria

Department of Electrical Engineering

University Center Nour Bachir of El-Bayadh

El-Bayadh, Algeria

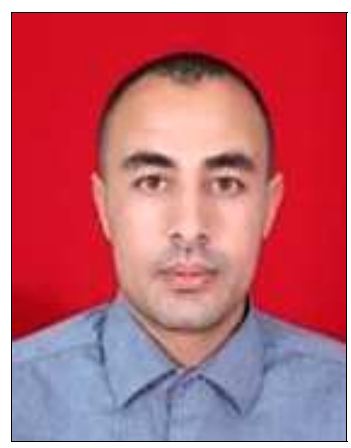

Mohamed Rida Lahcene received his B.Eng. degree in 2009, M.Sc. in 2012, and Ph.D. in 2018 - all from the University of Bechar Algeria. Since 2020, he has been working as an Associate Professor at the University Center Salhi Ahmed of Naama, Algeria. His main interests are in turbo encoding schemes, iterative decoding over fading channels, and complexity of encoders.

E-mail: lahcenreda1@gmail.com

Information Processing and Telecommunication

Laboratory (LTIT)

Tahri Mohammed Bechar University

Bechar, Algeria

Department of Technology

University Center Salhi Ahmed of Naama

Naama, Algeria

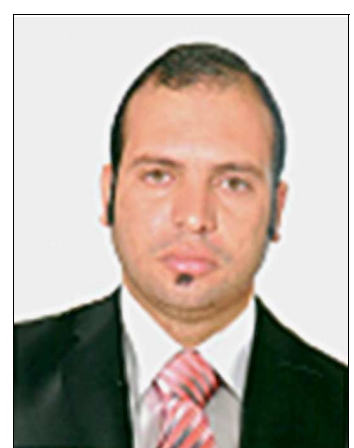

Fayssal Menezla obtained his B.Eng. degree in Telecommunications, M.Sc. in Electronics and Ph.D. in Electronics, all from University of Djillali Liabes of Sidi Bel Abbes, Algeria. His research interests are in image processing, source coding, channel codes, joint coding, wireless communication systems and optimal encoders.

E-mail: menezla@yahoo.fr Department of Electrical Engineering University Center Nour Bachir of El-Bayadh El-Bayadh, Algeria

Laboratory (LEPO)

Djillali Liabes University

Sidi Bel-Abbes, Algeria

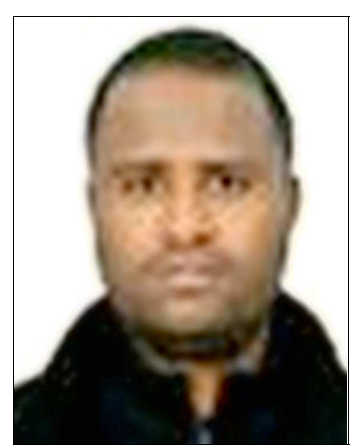

Elarbi Abderraouf received the B.Eng. degree from the University of Molay Taher Saida, Algeria in 2008, and his M.Sc. from the University of Bechar, Algeria in 2013, and the Ph.D. degree, in 2020, from University of Bechar, Algeria. His main interests are turbo encoding schemes, multicarrierCDMA transmission techniques for integrated broadband cellular systems, iterative decoding over fading channels.

E-mail: elarbiabderraouf12@gmail.com

Information Processing and Telecommunication

Laboratory (LTIT)

Tahri Mohammed Bechar University

Bechar, Algeria 